\title{
Experimentelle Studien über intravenöse Fettinfusion unter besonderer Berücksichtigung parenteraler Ernährung.
}

\author{
IV. Mitteilung. \\ Kraftwechsel nach Fettinfusion. \\ Von \\ Dr. Toshiharu Nomura. \\ (野 村 利 治) \\ (Aus der medizinischen Klinik on Prof. S. Yamakawa, \\ an der Universität zu Sendai.)
}

In der letzten Mitteilung habe ich festgestellt, dass direkt in die Blutbahn eingeführtes, fremdartiges Fett in den verschiedenen Körperteilen abgelagert werden kann. Was dann aus diesem abgelagerten Fettanteil wird, oder ob überhaupt direkt ins Blut eingeführte Fettkörper zum Stoffund Kraftwechsel richtig verwertet werden können, das ist die nächstliegende Frage, welche die Lehre von der parenteralen Ernährung zu lösen hat. v. Leu be, ${ }^{1)}$ der seinerzeit subkutane Fettzufuhr für parenterale Ernährung eifrig untersucht hat, führte schon einen grundlegenden Versuch aus, indem er bei einern lange mit magerem Fleisch ernährten und daher sehr fettarm gewordenen Hund nach wiederholten Butterinfusionen reichliche Fettablagerung am Unterhautfettgewebe und Mesenterium beobachtete, die aber nach abermaliger reiner Fleischernährung verschwand. Er zog aus diesem Ergebnis den Schluss, dass subkutan eingeführtes Fett auch richtig verwertet werden kann. $\mathrm{Zu}$ diesem Versuche benutzte aber v. Leube, wie bei den damals lebhaft empfohlenen therapeutischen Fettinfusionen (Jacob,, ${ }^{2}$ Du Mesnil,, Strauss ${ }^{4)}$ ), als Infusionsmaterial ausschliesslich nicht emulgiertes Fett, und dass der Nutzeffekt dieser Applikationsmethode infolge sehr langsamer Resorption recht mangelhaft ist, darauf wurde spüter von Winternitz, ${ }^{5)}$ Heilner ${ }^{(6)}$ u. a. nachdrücklich hingewiesen. Mills und Murlin injizierten Kaninchen eine Lezithin- $\mathbf{O}^{i-}$ emulsion subkutan und gaben an, dass sie auf diese Weise ungefähr 25 Prozent des Kalorienbedarfs zuführen und auf den N-Stoffwechsel des Tiers 
einen günstigen Einfluss ausüben konnten. Wenn man also zum Zweck parenteraler Ernährung Fettzufuhr vornehmen und dabei einen annühernd naturgetreuen Nutzeffekt erlangen will, so ist es unzweifelhaft nötig, als Infusionsmaterial eine so fein zerstäubte Erulsion zu wählen, wie man sie etwa beiru Chylus finden kann; und dieses Erfordernis erfüllt gerade unser ,Yanol."

Zunïchst gehe ich auf die Frage ein : Wird direkt ins Blut gebrachtes Fett sofort als Energiequelle verwertet? Un hierüber Aufklïrung zu erhalten, habe ich den Grundumsat $z$ der Versuchstiere auf indirektem Weg untersucht, um zu erkennen, ob die Verbrennung des injizierten Fetts, wenn sie überhaupt stattfindet, durch Zunahme der Fettzersetzung oder durch Senkung des respiratorischen Quotienten erfolgt.

Zu" diesem Versuche wurden ausscbliesslich mïnnliche Kaninchen ohne Narkose gebraucht und die Respirationsrersuche nach $\mathrm{Hald}$ a ne, ${ }^{8}$ der N-Wert des Harns nach K jel$\mathrm{d} a \mathrm{hl}$ bestimmt. Der in einer Zeiteinheit ausgeschiedene Harn wurde durch Katheterisierung quantitativ aufgefangen. Aus den erhaltenen Analysenwerten wurde zuerst der Wert des Respirationsquotienten ( $R$.-Q.), der sich nicht auf Proteine bezieht, ermittelt und daraus nach Zuntz und Schumburg ${ }^{\text {i) }}$ weiter die im Körper zersetzte Menge an Eiweiss, Fett und Kohlehydrat ausgerechnet.

Zuerst bemühte ich mich un die Bestimmung der kürzesten, zur Erlangung der Gaswechsel werte hinreichenden Versuchsdauer. Dazu wurden anfangs die Respirationsversuche unter gleichen Bedingungen rerschieden lange (1-6 Stunden) fortgefiihrt, es fand sich aber in den Ergebnissen kein merklicher Cnterschied. Deshalb habe ich in diesen Versuche die Dauer des Respirationsrersuchs gewöhnlich auf 1-2 Stunden beschrünkt, solange die gleichmiissige Bedingung durch Fettinfusion nicht ge:̈ndert war. Während dieser Zeiteinheit wurden für die Ventilation 3 Liter Luft pro Minute nach $\mathrm{M}$ a r in $\mathrm{e}^{10}$ ) durchgetrieben. Die Kaninchen wurden ror dem eigentlichen Versuche mindestens eine Woche im Versuchszimmer mit gleichmüssiger Nahrung gefüttert, wodurch der Gaswechsel der Tiere auf eine fast konstante Grösse gebracht wurde. Dann wurde an den darauffolgenden 5 Tagen in der letzten Stunde ror der Fiitterung jedesmal ein Respirationsversuch ausgeführt, um aus diesen Leerversuchen die Gaswechselgrösse des nüchternen Tiers zu erhalten. Am 6ten Versuchstage, an dem erst der Hauptrersuch anzustellen war, wurde ein Leerversuch wie gewöhnlich porausgeschickt, aber im Anschluss daran erfolgte keine Fütterung, sondern $10 \mathrm{ccm}$ „Yanol" pro kg Gewicht wurden intrarenös eingespritzt, und während den darauffolgenden 5 Stunden je 1 Respirationsversuch und Harnanalyse ausgeführt, um irgendwelche Schwankungen des Grundumsatzes, die rielleicht durch Fettinfusion verursacht worden sein könnten, zu verfolgen.

Wie die Tabellen zeigen, ergibt sich während 5 Stunden nach Fettinfusion bezïglich der Kalorienbildung im Vergleich mit den Leerversuchen fast keine Veränderung. Ferner liess sich in diesem Versuch nach Fettinfusion keine Senkung des R.-Q. resp. Zunahme der Fettzersetzung nachweisen, was sich, wenn das infundierte Fett als überschüssig zugeführtes Material schnell verbrannt würde, leicht erwarten liesse. Kleine Schwankungen in den Ergebnissen liegen vielmehr innerhalb der Sthwankungs- 
Tabelle I.

Grundumsatz nach Fettinfusion.

\begin{tabular}{|c|c|c|c|c|c|c|c|c|c|c|c|c|}
\hline & $\underset{\Xi}{\vec{\Xi}}$ & $\underset{0}{5}$ & 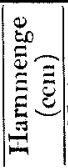 & 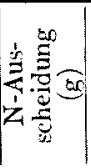 & $\begin{array}{l}30 \\
0 \\
0 \\
\text { (pro }\end{array}$ & 8 & $\begin{array}{c}\dot{\leftrightarrow} \\
\dot{\epsilon}\end{array}$ & 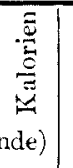 & 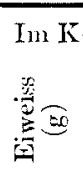 & $\frac{3}{\stackrel{3}{3}}$ & 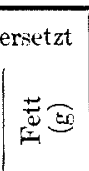 & 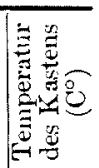 \\
\hline 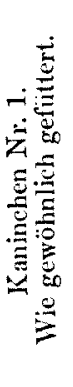 & 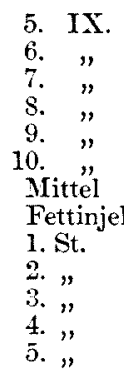 & $\begin{array}{c}1803,27 \\
1787,00 \\
1758,30 \\
1749,95 \\
1745,09 \\
1710,25 \\
1758,97 \\
\text { tion } 9 \text { Ub } \\
1728,49 \\
1715,95 \\
1707,29 \\
1700,32 \\
1694,89\end{array}$ & $\begin{array}{l}5,2 \\
4,0 \\
3,4 \\
4,4 \\
4,1 \\
2,8 \\
3,9 \\
\text { a.m } \\
5,0 \\
5,2 \\
3,4 \\
3,6 \\
3,8\end{array}$ & $\begin{array}{l}0,024 \\
0,020 \\
0,020 \\
0,025 \\
0,024 \\
0,027 \\
0,023 \\
10 . \mathrm{I} \\
0,024 \\
0,025 \\
0,029 \\
0,020 \\
0,020\end{array}$ & $\begin{array}{l}0,89 \\
0,90 \\
0,94 \\
0,87 \\
0,86 \\
0,85 \\
0,88 \\
\text {. Sei } \\
0,85 \\
0,86 \\
0,87 \\
0,87 \\
0,88\end{array}$ & $\begin{array}{l}1,03 \\
1,11 \\
1,07 \\
1,00 \\
1,00 \\
0,95 \\
1,03 \\
1,24 \\
0,97 \\
1,00 \\
1,04 \\
1,04 \\
1,05\end{array}$ & $\begin{array}{l}0,85 \\
0,91 \\
0,83 \\
0,84 \\
0,86 \\
0,80 \\
0,85 \\
\text { Stund } \\
0,88 \\
0,85 \\
0,88 \\
0,87 \\
0,87\end{array}$ & $\begin{array}{l}2,98 \\
3,05 \\
3,15 \\
2,89 \\
2,87 \\
2,52 \\
2,96 \\
\text { den ke } \\
2,82 \\
2,86 \\
2,962 \\
2,92 \\
2,95 \\
2,98\end{array}$ & $\begin{array}{c}0,152 \\
0,129 \\
0,130 \\
0,156 \\
0,155 \\
0,168 \\
0,148 \\
\text { eine Fi } \\
0,150 \\
0,156 \\
0,181 \\
0,125 \\
0,125\end{array}$ & $\begin{array}{c}0,306 \\
0,467 \\
0,293 \\
0,271 \\
0,309 \\
0,180 \\
0,304 \\
\text { tterung } \\
0,247 \\
0,287 \\
0,340 \\
0,361 \\
0,364\end{array}$ & $\begin{array}{l}0,129 \\
0,082 \\
0,162 \\
0,131 \\
0,113 \\
0,154 \\
0,128\end{array}$ & $\begin{array}{c}29-30 \\
29 \\
30 \\
30 \\
27-28 \\
30 \\
27-30\end{array}$ \\
\hline 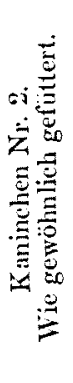 & 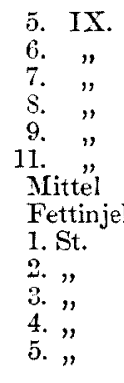 & $\begin{array}{l}44 \\
00 \\
25 \\
98 \\
14 \\
84 \\
61 \\
\text { Uh } \\
25 \\
55 \\
33 \\
74 \\
40\end{array}$ & $\begin{array}{l}4,0 \\
3,3 \\
3,6 \\
4,4 \\
6,2 \\
4,8 \\
4,4 \\
\text { a.m } \\
4,7 \\
2,0 \\
1,3 \\
0,3 \\
0,5\end{array}$ & $\begin{array}{l}0,029 \\
0,026 \\
0,028 \\
0,034 \\
0,032 \\
0,020 \\
0,028 \\
11 . \mathrm{I} \\
0,018 \\
0,018 \\
0,016 \\
0,012 \\
0,010\end{array}$ & $\begin{array}{l}0,93 \\
0,90 \\
0,93 \\
0,90 \\
0,93 \\
0,98 \\
0,93 \\
\text { X. Sei } \\
0,97 \\
0,97 \\
0,97 \\
0,98 \\
0,98\end{array}$ & $\begin{array}{l}1,12 \\
1,03 \\
1,10 \\
1,04 \\
1,06 \\
1,16 \\
1,08 \\
12,24 \\
1,14 \\
1,09 \\
1,15 \\
1,13 \\
1,14\end{array}$ & $\begin{array}{l}0,89 \\
0,83 \\
0,87 \\
0,85 \\
0,83 \\
0,86 \\
0,85 \\
\text { Stund } \\
0,86 \\
0,82 \\
0,86 \\
0,83 \\
0,89\end{array}$ & $\begin{array}{l}\mid \begin{array}{l}3,17 \\
3,02 \\
3,13 \\
3,01 \\
3,07 \\
3,32 \\
3,12\end{array} \\
\text { den ke }\end{array}$ & $\begin{array}{l}0,181 \\
0,162 \\
0,175 \\
0,212 \\
0,199 \\
0,125 \\
0,176 \\
\text { eine Fi } \\
0,112 \\
0,112 \\
0,100 \\
0,075 \\
0,062\end{array}$ & $\begin{array}{c}0,400 \\
0,262 \\
0,355 \\
0,274 \\
0,251 \\
0,389 \\
0,322 \\
\text { terung } \\
0,389 \\
0,285 \\
0,399 \\
0,334 \\
0,485\end{array}$ & $\begin{array}{l}0,181 \\
0,147 \\
0,184 \\
0,117\end{array}$ & $\begin{array}{c}30 \\
30 \\
28-29 \\
30 \\
27-28 \\
26-27 \\
26-30 \\
\\
26 \\
26-27 \\
26-27 \\
27 \\
27-28\end{array}$ \\
\hline 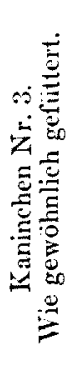 & 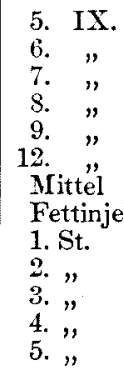 & $\begin{array}{l}48 \\
73 \\
, 38 \\
02 \\
15 \\
82 \\
93 \\
\text { Uh } \\
72\end{array}$ & $\begin{array}{r}2,2 \\
2,8 \\
2,7 \\
3,1 \\
2,8 \\
7,2 \\
3,5 \\
\text { a.m } \\
5,4 \\
3,7 \\
3,7 \\
3,2 \\
2,3\end{array}$ & $\begin{array}{l}0,017 \\
0,020 \\
0,026 \\
0,023 \\
0,026 \\
0,029 \\
0,024 \\
12 . \mathrm{II} \\
0,017 \\
0,026 \\
0,028 \\
0,027 \\
0,020\end{array}$ & $\begin{array}{l}0,92 \\
0,87 \\
0,90 \\
0,93 \\
0,97 \\
0,94 \\
0,92\end{array}$ & $\begin{array}{l}1,11 \\
1,03 \\
1,08 \\
1,09 \\
1,15 \\
1,11\end{array}$ & $\begin{array}{l}0,89 \\
0,86 \\
0,87 \\
0,85 \\
0,86 \\
0,87 \\
0,87 \\
\text { Stund } \\
0,86 \\
0,81 \\
0,85 \\
0,89 \\
0,87\end{array}$ & $\begin{array}{l}3,12 \\
2,94 \\
3,04 \\
3,12 \\
3,27 \\
3,15 \\
3,11 \\
\text { den ke } \\
3,20 \\
3,21 \\
3,12 \\
3,01 \\
3,02\end{array}$ & $\begin{array}{c}0,106 \\
0,125 \\
0,162 \\
0,143 \\
0,162 \\
0,181 \\
0,146 \\
\text { ceine F }\end{array}$ & $\begin{array}{l}0,446 \\
0,335 \\
0,350 \\
0,328 \\
0,366 \\
0,354 \\
0,363\end{array}$ & $\begin{array}{l}0,100 \\
0,092 \\
0,118\end{array}$ & $\begin{array}{c}28 \\
28 \\
28-28 \\
28 \\
28 \\
27 \\
27-25 \\
\\
27 \\
29-30 \\
29-30 \\
29-30 \\
29\end{array}$ \\
\hline
\end{tabular}

breite der Versuche, denn man kann solche auch bei Infusion physiologischer Kochsalzlösung beobachten, wie aus nebenstehenden Tabellen ersichtlich ist. 


\section{Tabelle II.}

Grundumsatz nach Kochsalzinfusion.

Kaninchen $\mathrm{Nr} .4$. Wie gewöhnlich gefütert.

$10 \mathrm{~cm} 0,9 \%$ iger Kochsalzlösung pro $\mathrm{kg}$ i.v.

\begin{tabular}{|c|c|c|c|c|c|c|c|c|c|c|c|}
\hline & & & & bo & 30 & $\varepsilon$ &. & \multicolumn{3}{|c|}{ Im Körper zersetzt } & \multirow{2}{*}{ 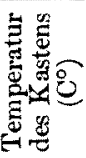 } \\
\hline$\stackrel{\Xi}{E}$ & 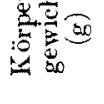 & 烝 & 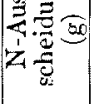 & $\begin{array}{l}0^{-9} \\
(\mathrm{pr}\end{array}$ & $\delta^{8}$ & Stun & e) & $\frac{200}{30}$ & 离包 & 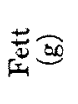 & \\
\hline 25. & & 2,0 & 0 & 0 & 1,00 & & 2,86 & 0,145 & 0,336 & 0,107 & 30 \\
\hline 26. & & 3,2 & 0,027 & 0,81 & 0,92 & & 2,70 & 0,168 & 0,206 & 0,130 & $29-$ \\
\hline & & 12,0 & 6 & 0 & 1,06 & & 2,9 & 0,1 & 0, & 0 & 29 \\
\hline & & & & 0,92 & 1,09 & & $\overrightarrow{3}$, & 0,1 & & & \\
\hline 29 . & & 11,2 & 0,0 & 0,85 & 0,99 & 0 & 2,87 & 0,176 & 0,275 & & 29 \\
\hline & & & & & 1,02 & & 3 & & & & 30 \\
\hline Mitte & & 6 & & & 1, & & 2, & 0,169 & & & \\
\hline \multicolumn{12}{|c|}{ Kochsalzinjektion 9} \\
\hline & & & & & 3,05 & & & 0,1 & & & $1-$ \\
\hline & & 1 & & & 1 & & & 3 & & & $32-3$ \\
\hline & & 1 & & 0 & 0,93 & & 2 & & & & 33 \\
\hline & & 1 & & & 1 & & 2 & & & & $33-34$ \\
\hline \multirow{2}{*}{\multicolumn{12}{|c|}{ Dasselbe Versuchstier. Am Aten FIungertag. }} \\
\hline & & & & & & & & & & & \\
\hline & & 1,2 & & & 0,30 & 0,71 & 2,56 & 0,150 & 0,007 & 204 & $31-32$ \\
\hline \multicolumn{12}{|c|}{ jektion 9} \\
\hline & & 1,8 & & 0,77 & 0,83 & & 2,51 & & & & $30-3$ \\
\hline & & & & & 0 & & 2, & & & & 32 \\
\hline & & I. & & & 0 & & 2,4 & & & & $32-33$ \\
\hline & & 1 , & & & 0,75 & & 2,38 & & 0, & & 32 \\
\hline 5. " & 1421,03 & 1,5 & 0,021 & 0,74 & 0,76 & 0,72 & 2,42 & 0,131 & 0,024 & 0,192 & 32 \\
\hline
\end{tabular}

Aus diesem negativen Ergebnis darf man aber keineswegs schliessen, dass das infundierte Fett überhaupt nicht verbrannt würde, denn die Fettzersetzung im Körper ging, wie man aus den Tabellen sieht, nach Fettinfusion genau wie vorher kontinuierlich vor sich, wobei natürlich nicht ausgeschlossen ist, dass das infundierte Fett auch daran beteiligt war. Um die Zeit, in der die Infusionsversuche vorgenommen wurden, waren die Versuchstiere, die sich nur im nüchternen Zustande befanden und nicht lange gehungert hatten, noch mit genügender Menge Kohlehydratvorrat versehen, und deshalb ging der Kraftwechsel wie gewöhnlich mit Kohlehydrat als hauptsächlichem Energiespender vor sich, wodurch allerdings keineswegs gesteigerte Fettzersetzung hervorgerufen werden muss, selbst wenn diese Substanz reichlich zur Verfügung steht.

$\mathrm{Da}$ in den obigen Versuchen infolge genügenden Kohlehydratvorrats keine Vermehrung der Fettverbrennung herbeigeführt werden konnte, liess ich in der zweiten Versuchsreihe dieselben Versuchstiere des ersten Ver- 
suchs solange hungern, bis die Kohlehydratdepots grösstenteils erschöpft waren. Am 4ten Hungertage wurde in der letzten Stunde vor der Fettinfusion ein Leerversuch wie gewöhnlich vorausgeschickt und dann der Hauptversuch ebenso wie bei der vorigen Versuchsreihe ausgeführt.

\section{Tabelle III.}

Grundumsatz nach Fettinfusion bei müssiger Karenz.

\begin{tabular}{|c|c|c|c|c|c|c|c|c|c|c|c|c|}
\hline & $\underset{\Xi}{\Xi}$ & 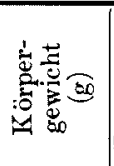 & 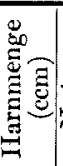 & 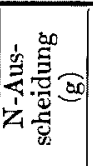 & $\left|\begin{array}{c}0 \\
0 \\
0 \\
\text { (pro }\end{array}\right|$ & $\begin{array}{c}30 \\
8 \\
8 \\
8\end{array}$ & 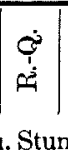 & 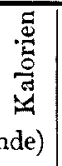 & 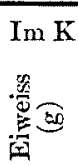 & 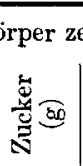 & 吾 & 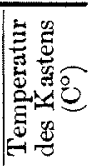 \\
\hline 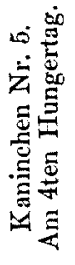 & $\begin{array}{l}\text { Letzte St. } \\
\text { vor d. Inj. } \\
\text { Fettinjek } \\
\text { 1. St. } \\
2 . ", \\
3 . ", \\
4 . ", \\
5 . ",\end{array}$ & $\begin{array}{r}1584,43 \\
\text { ion } 9 \text { Uh } \\
1599,12 \\
1582,92 \\
1577,30 \\
1574,68 \\
1571,74\end{array}$ & $\begin{array}{r}1,0 \\
\text { r a.m } \\
2,4 \\
2,7 \\
1,0 \\
1,0 \\
0,7\end{array}$ & $\begin{array}{l}0,020 \\
13 . \mathrm{IX} \\
0,01 \mathrm{X} \\
0,023 \\
0,023 \\
0,017 \\
0,015\end{array}$ & $\begin{array}{l}0,85 \\
x . \\
0,82 \\
0,85 \\
0,85 \\
0,84 \\
0,85\end{array}$ & $\begin{array}{l}0,89 \\
0,86 \\
0,90 \\
0,91 \\
0,87 \\
0,87\end{array}$ & $\begin{array}{l}0,75 \\
0,75 \\
0,75 \\
0,77 \\
0,74 \\
0,72\end{array}$ & $\begin{array}{l}2,64 \\
2,69 \\
2,79 \\
2,80 \\
2,76 \\
2,79\end{array}$ & $\begin{array}{l}0,125 \\
0,112 \\
0,143 \\
0,143 \\
0,106 \\
0,093\end{array}$ & $\begin{array}{l}0,088 \\
0,088 \\
0,087 \\
0,127 \\
0,071 \\
0,030\end{array}$ & \begin{tabular}{l|}
0,203 \\
\\
0,202 \\
0,200 \\
0,184 \\
0,220 \\
0,245
\end{tabular} & $\begin{array}{c}29-30 \\
30 \\
30-31 \\
31 \\
30-31 \\
31\end{array}$ \\
\hline 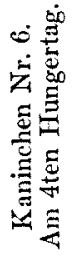 & $\begin{array}{l}\text { Letzte St. } \\
\text { vor d. Inj. } \\
\text { Fettinjek } \\
\text { I. St. } \\
2 . \text { ", } \\
3 . \text { " } \\
4 . \text { " } \\
5 . \text { " }\end{array}$ & $\begin{array}{l}62 \\
\text { Uh } \\
08 \\
28 \\
83 \\
55 \\
39\end{array}$ & $\begin{array}{l}1,5 \\
\text { a.m } \\
1,4 \\
1,3 \\
1,7 \\
1,4 \\
1,3\end{array}$ & $\begin{array}{l}0,027 \\
14 . \mathrm{IX} \\
0,023 \\
0,025 \\
0,026 \\
0,028 \\
0,024\end{array}$ & $\begin{array}{l}0,86 \\
0,87 \\
0,89 \\
0,83 \\
0,85\end{array}$ & $\begin{array}{l}0,91 \\
0,94 \\
0,97 \\
0,90 \\
0,90\end{array}$ & $\begin{array}{l}0,76 \\
0,78 \\
0,77 \\
0,77 \\
0,76\end{array}$ & $\begin{array}{l}2,78 \\
2,82 \\
2,86 \\
2,95 \\
2,73 \\
2,79\end{array}$ & $\begin{array}{l}0,168 \\
0,143 \\
0,156 \\
0,162 \\
0,115 \\
0,150\end{array}$ & $\begin{array}{l}0,138 \\
0,108 \\
0,147 \\
0,131 \\
0,175 \\
0,105\end{array}$ & $\begin{array}{l}0,166 \\
0,194 \\
0,177 \\
0,190 \\
0,167 \\
0,189\end{array}$ & $\begin{array}{c}25-27 \\
\\
27 \\
27-28 \\
27-28 \\
28 \\
28\end{array}$ \\
\hline 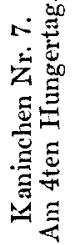 & $\begin{array}{l}\text { Letzte St. } \\
\text { vor d. Inj. } \\
\text { Fettinjek } \\
\text { 1. St. } \\
2 . \text { " } \\
\text { 3. "" } \\
\text { 4. ", } \\
\text { 5. ", }\end{array}$ & $\begin{array}{r}1494,73 \\
\text { ion } 9 \text { Uh } \\
1517,86 \\
1500,95 \\
1495,70 \\
1490,05 \\
1485,70\end{array}$ & $\begin{array}{r}1,2 \\
\text { r a.m } \\
2,9 \\
2,5 \\
2,1 \\
2,1 \\
1,3\end{array}$ & $\begin{array}{l}0,025 \\
15 . \mathrm{IX} \\
0,029 \\
0,018 \\
0,024 \\
0,024 \\
0,021\end{array}$ & $\begin{array}{l}0,89 \\
\mathrm{X} . \\
0,93 \\
0,94 \\
0,91 \\
0,90 \\
0,91\end{array}$ & $\begin{array}{l}0,99 \\
0,10 \\
0,98 \\
0,95 \\
0,99\end{array}$ & $\mid \begin{array}{l}0,75 \\
0,78 \\
0,77 \\
0,75 \\
0,78\end{array}$ & $\begin{array}{l}2,92 \\
3,05 \\
3,10 \\
2,99 \\
2,94 \\
3,02\end{array}$ & $\begin{array}{l}0,156 \\
0,181 \\
0,112 \\
0,150 \\
0,150 \\
0,131\end{array}$ & $\begin{array}{l}0,131 \\
0,191 \\
0,177 \\
0,137 \\
0,092 \\
0,165\end{array}$ & $\begin{array}{l}0,190 \\
0,208 \\
0,212 \\
0,200 \\
0,211 \\
0,200\end{array}$ & $\begin{array}{c}26-27 \\
27-28 \\
27-28 \\
29 \\
28-29 \\
28-29\end{array}$ \\
\hline
\end{tabular}

Vergleicht man zunïchst das Ergebnis der Leerversuche mit dem der vorigen Versuchsreihe, so ersieht man, dass als Folge der Karenz das Fett jetzt als dominierende Energiequelle leblaft verbrennt, wodurch der R.-Q. deutlich verkleinert wird. Die N-Ausscheidung ist auch als Zeichen fortschreitender Karenz stark vermindert. Nach Fettinfusion wurden aber diese Verbältnisse immer auch bei dieser Versuchsreihe nicht merklich verändert. Nur soviel liess sich dabei beobachten, dass die Zersetzung des Fetts ein bisschen gesteigert, die des Eiweisses dementsprechend vermindert wurde. 
Diese kleine Schwankung geht indessen nur wenig über die Fehlergrenze hinaus, wenn sie auch bei allen Versuchsfällen konstant war. Jedenfalls wurde durch dieses Ergebnis das eine sichergestellt, dass direkt infundiertes Fett auch bei Hunger keine nennenswerte Steigerung der Fettzersetzung veranlasst. Dies ist aber kein Wunder, wenn man daran denkt, dass bei Hunger, solange der Körper noch genïgend Fett hat, diese Substanz als Hauptquelle der Energie in Anspruch genommen wird, und wenn auch in diesem Stadium eine Menge Fett in die Zirkulation eingeführt wird, dies doch keine Steigerung des Fettzerfalls zur Folge zu haben braucht, denn Fett übt bekanntlich auf den Stoffwechsel keine spezifisch-dynamische Wirkung aus. Erst dann liesse sich also von einem direkten Beweis für die Verbrennung des infundierten Fetts sprechen, wenn der Fettvorrat durch eine noch tiefer greifende Karenz vom Körper sehr verschleppt würde und die nummehr eintretende Zersetzung des Körpereiweisses, das als letzter Energiespender mobilisiert würde, durch Fettinfusion zurückgehalten werden könnte.

Das Ergebnis derartigen Versuche sei im Folgenden angeführt. In diesen Versuche liess ich das Kaninchen 6 resp. 8 Tage lang hungern. Nach diesen Karenztagen hatte sich der Fettzerfall deutlich herabgesetzt. Am 7resp. 9ten Hungertage erhielt das Tier $20 \mathrm{ccm}$ "Yanol" intravenös. Die Respirationsversuche, die in den übrigen Hungertagen nur 2 Stunden lang ausgeführt worden waren, wurden direkt nach der Fettinfusion auf 18 Stunden ausgedehnt.

Wie man aus der Tabelle ersieht, blieb die Fettzersetzung in den ersten Stunden nach Fettinfusion wie vorher gleich gross, aber von etwa 3. oder 4. Stunde an wurde sie allmïhlich immer lebhafter, wobei der Eiweisszerfall im Gegensatz dazu sich merklich verkleinerte. Eine noch längere Beobachtung, die weitere Karenztage erfordert, konnten leider die kleinen Versuchstiere wegen der schon eingetretenen hochgradigen Erschöpfung nicht mehr vertragen.

Aus den oben angeführten Versuchen wurde festgestellt, dass direkt eingespritztes Fett niemals übermüssige Fettzersetzung im Körper hervorruft, sondern ruhig zum Stoffwechsel verwertet wird; eine Eigenschaft, die vor allen Dingen dem Fett den besten Charakter als parenteralem Nährstoff ausstellt. Das ist heute noch eine Streitfrage, ob Fett als solches direkt verbrennt oder erst auf dem Umweg über den Zucker als Energiequelle dienen kann. Letztere Ansicht wurde früher von manchen vertreten, wird aber heute besonders von amerikanischen Autoren abgelehnt, die meinen, dass Fett wenigstens beim Fehlen von Produkten des Kohlehydratstoff- 


\section{Tabelle IV.}

Grundumsatz nach Fettinfusion bei fortgeschrittener Karenz.

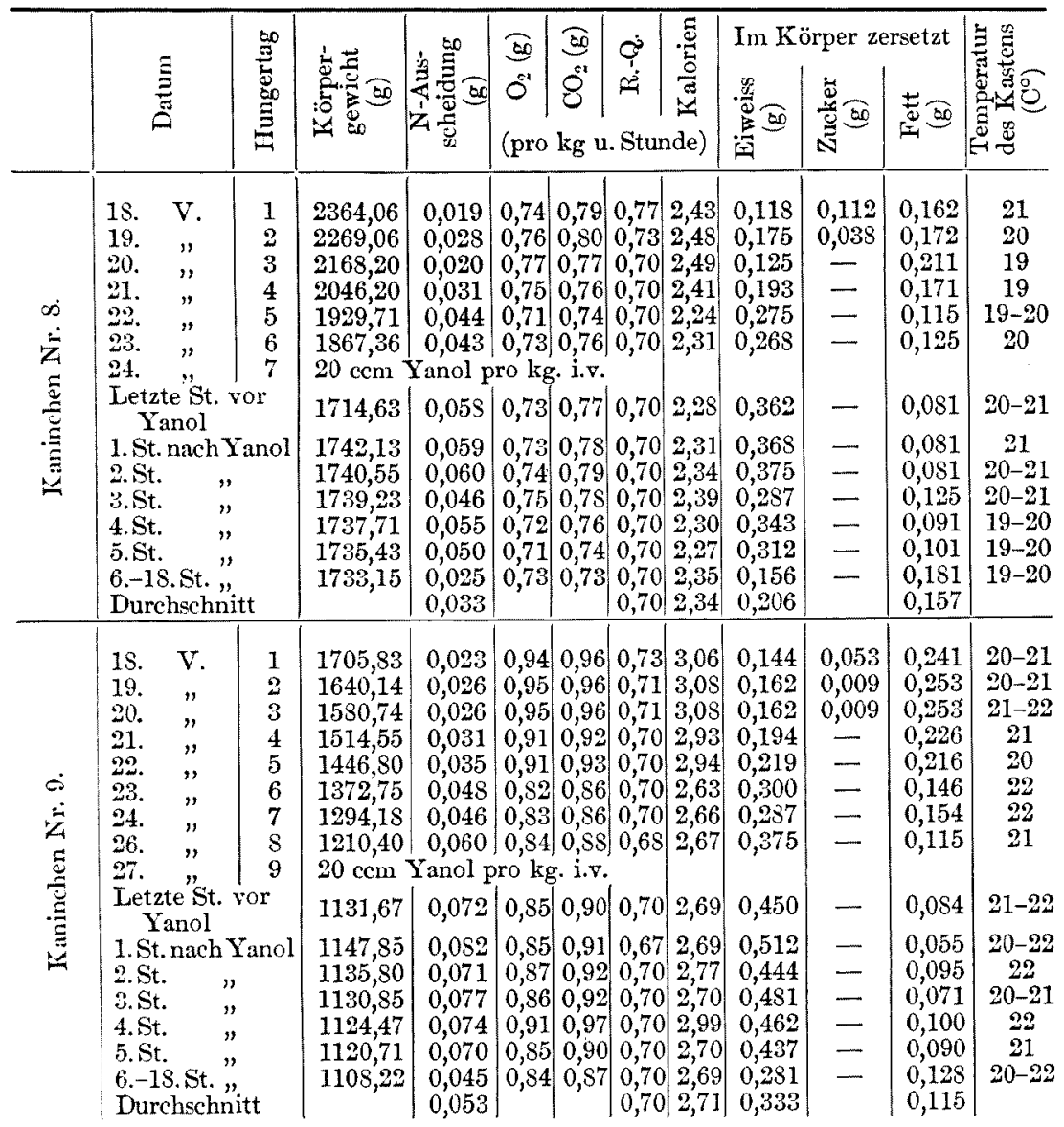

wechsels ohne vorherige Umwandlung in Zucker zur Muskelarbeit oxydiert und benutzt werden kann. Jedenfalls wurde in meinem Versuche beobachtet, dass Fett bei parenteraler Zufuhr auch erst langsam verbrennt. In dieser Beziehung wäre es auch von Interesse zu prüfen, was denn mit dem in die Blutbahn gelangten Zucker geschieht. Es gehört nicht zu meinem eigentlichen Thema, auf die Frage der Zuckerzersetzung im Körper einzugehen. Indessen kann Zucker, der im normalen Verhültnis der hauptsïchliche Energiespender ist, bei dieser Versuchsanordnung ein ganz anderes Ergebnis bieten, das vielleicht als gute Kontrolle für das beim Fett 
beobachtete dienen dürfte. Dass intravenös einverleibter Traubenzucker zum 'Teil kurz nach der Injektion verbrennt, darauf haben schon Falta

\section{Tabelle V.}

Grundumsatz nach Zuckerinfusion.

\begin{tabular}{|c|c|c|c|c|c|c|c|c|c|c|c|c|}
\hline & $\underset{\Xi}{\tilde{\Xi}}$ & 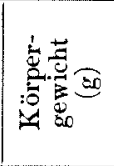 & 题 & 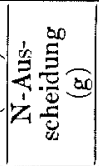 & $\left|\begin{array}{c}30 \\
0 \\
0 \\
\text { (pro }\end{array}\right|$ & $\left|\begin{array}{c}80 \\
8 \\
8\end{array}\right|$ & $\begin{array}{c}\dot{Y} \\
\dot{q}\end{array}$ & 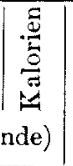 & 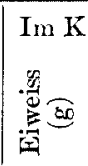 & $\stackrel{\vec{y}}{\vec{y}}$ & 表 & 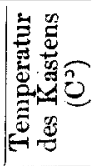 \\
\hline 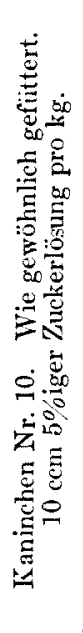 & $\begin{array}{l}\text { 7. VIII. } \\
8 . ", \\
9 . ", \\
10 . ", \\
11 . ", \\
12 . ", \\
\text { Mittel } \\
\text { Zuckerinj } \\
\text { 1. St. } \\
2 ., " \\
3 . ", \\
4 . " \\
5 . " \\
\text { Dasselbe }\end{array}$ & \begin{tabular}{|}
1680,65 \\
1717,79 \\
1688,08 \\
1678,97 \\
1731,66 \\
1699,14 \\
1699,38 \\
ektion 9 \\
1712,00 \\
1702,59 \\
1691,59 \\
1684,33 \\
1676,57 \\
Versuchst
\end{tabular} & \begin{tabular}{|}
1,8 \\
3,7 \\
1,9 \\
4,0 \\
4,2 \\
2,2 \\
3,0 \\
Uhr a \\
2,3 \\
4,8 \\
2,2 \\
2,8 \\
2,4
\end{tabular} & $\begin{array}{l}0,026 \\
0,016 \\
0,020 \\
0,024 \\
0,026 \\
0,020 \\
0,022\end{array}$ & $\begin{array}{l}0,98 \\
0,98 \\
0,96 \\
0,93 \\
0,93 \\
0,98 \\
0,96 \\
\text { VIII } \\
0,93\end{array}$ & $\begin{array}{l}1,17 \\
1,10 \\
1,13 \\
1,12 \\
1,05 \\
1,15 \\
1,12 \\
\text { I. Seit } \\
\mid 1,14 \\
1,15 \\
1,02 \\
1,06 \\
0,99\end{array}$ & $\begin{array}{l}0,88 \\
0,81 \\
0,87 \\
0,89 \\
0,82 \\
0,86 \\
0,85\end{array}$ & $\begin{array}{l}3,29 \\
3,27 \\
3,22 \\
3,13 \\
3,08 \\
3,26 \\
3,21\end{array}$ & $\begin{array}{c}0,162 \\
0,100 \\
0,125 \\
0,150 \\
0,162 \\
0,125 \\
0,137 \\
\text { len kein } \\
0,137 \\
0,156 \\
0,168 \\
0,156 \\
0,168\end{array}$ & $\begin{array}{l}0,410 \\
0,296 \\
0,400 \\
0,417 \\
0,247 \\
0,382 \\
0,359 \\
e \text { Fuitte } \\
0,455 \\
0,448 \\
0,396 \\
0,274 \\
0,227\end{array}$ & \begin{tabular}{|l|}
0,104 \\
0,199 \\
0,127 \\
0,101 \\
0,158 \\
0,139 \\
0,138
\end{tabular} & $\begin{array}{c}27-29 \\
28 \\
27-28 \\
28-29 \\
29 \\
28\end{array}$ \\
\hline 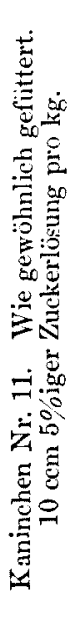 & $\begin{array}{l}\text { 7. VIII. } \\
\text { 8. " } \\
9 . \text { " } \\
10 . ", \\
11 . ", \\
13 . \text { " } \\
\text { Mittel } \\
\text { Zuckerinj } \\
1 . \text { St. } \\
2 . " \\
3 . " \\
4 . " \\
5 . " \\
\text { Dasselbe }\end{array}$ & $\begin{array}{l}185 \\
185 \\
185\end{array}$ & $\begin{array}{l}3,8 \\
5,2 \\
2,0 \\
1,3 \\
5,6 \\
2,0 \\
3,3\end{array}$ & $\begin{array}{l}0,028 \\
0,021 \\
0,023 \\
0,020 \\
0,020 \\
0,026 \\
0,023\end{array}$ & \begin{tabular}{|l|}
0,82 \\
0,87 \\
0,84 \\
0,87 \\
0,86 \\
0,89 \\
0,86 \\
VIII \\
0,91 \\
0,88 \\
0,88 \\
0,86 \\
0,88
\end{tabular} & $\begin{array}{l}1,07 \\
1,06 \\
1,01 \\
1,02 \\
1,03 \\
1,04 \\
1,04 \\
\text { I. Seit } \\
\left|\begin{array}{l}1,10 \\
1,09 \\
0,98 \\
0,95 \\
0,98\end{array}\right|\end{array}$ & $\begin{array}{l}0, S 5 \\
0,90 \\
0,89 \\
0,85 \\
0,88 \\
0,85 \\
0,87\end{array}$ & $\mid \begin{array}{l}3,08 \\
2,96 \\
2,94 \\
2,90 \\
2,92 \\
2,99 \\
2,95\end{array}$ & $\begin{array}{l}0,175 \\
0,131 \\
0,143 \\
0,125 \\
0,125 \\
0,162 \\
0,143\end{array}$ & $\begin{array}{l}0,305 \\
0,424 \\
0,372 \\
0,310 \\
0,355 \\
0,300 \\
0,344\end{array}$ & $\begin{array}{l}162 \\
267 \\
264 \\
249\end{array}$ & $\begin{array}{c}28-29 \\
27-28 \\
28 \\
28-29 \\
29 \\
29-30 \\
27-30 \\
\\
29-30 \\
30 \\
30-31 \\
30-31 \\
31-32\end{array}$ \\
\hline
\end{tabular}




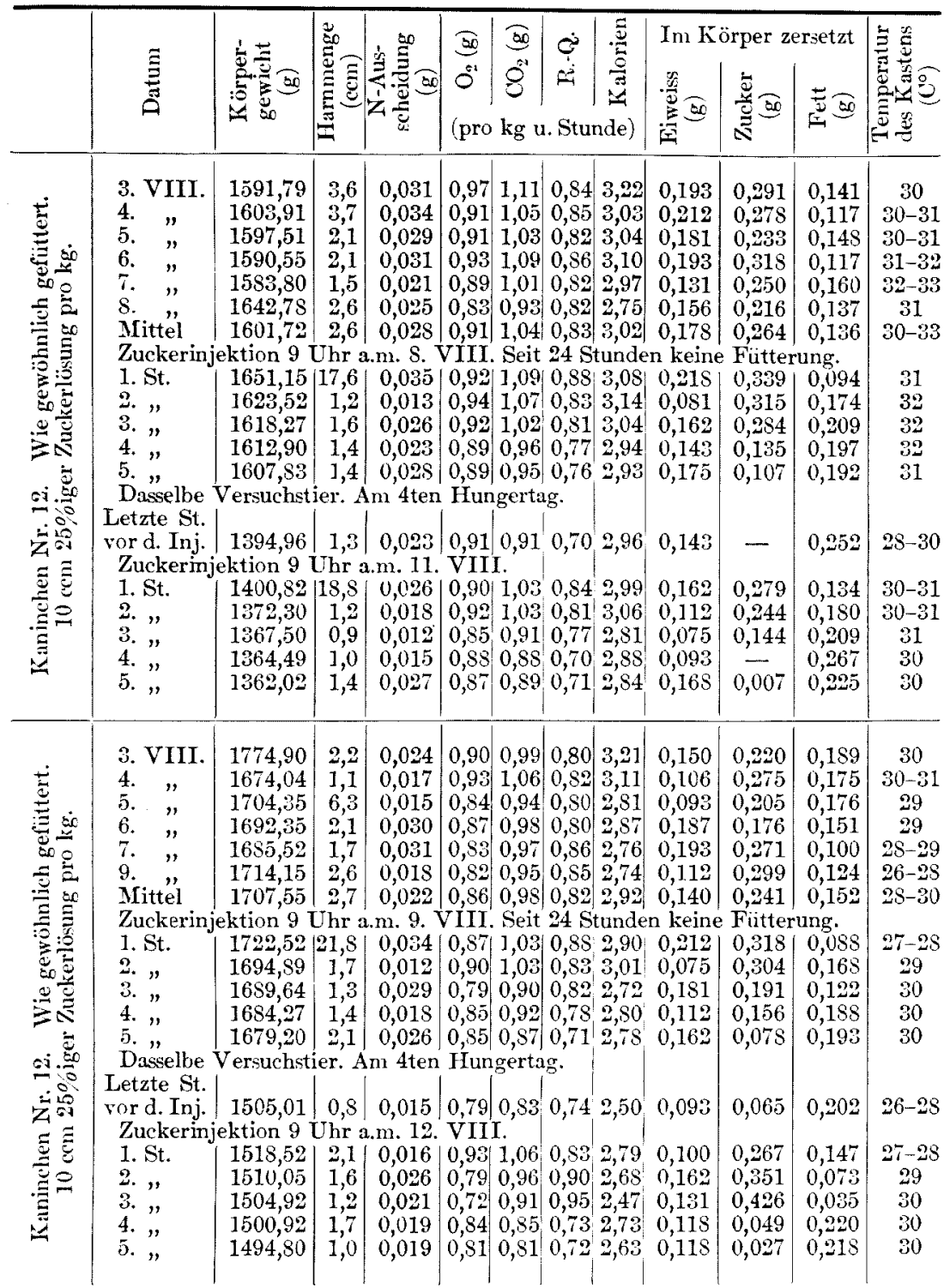

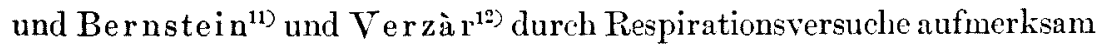
gemacht. Verzàr beobachtete das Anwachsen des R.-Q. im Augenblick der Zuckerinfusion, während die ersteren diese Erscheinung erst einige Stunden danach fanden. Diese Meinungsverschiedenheit zwischen den Autoren 


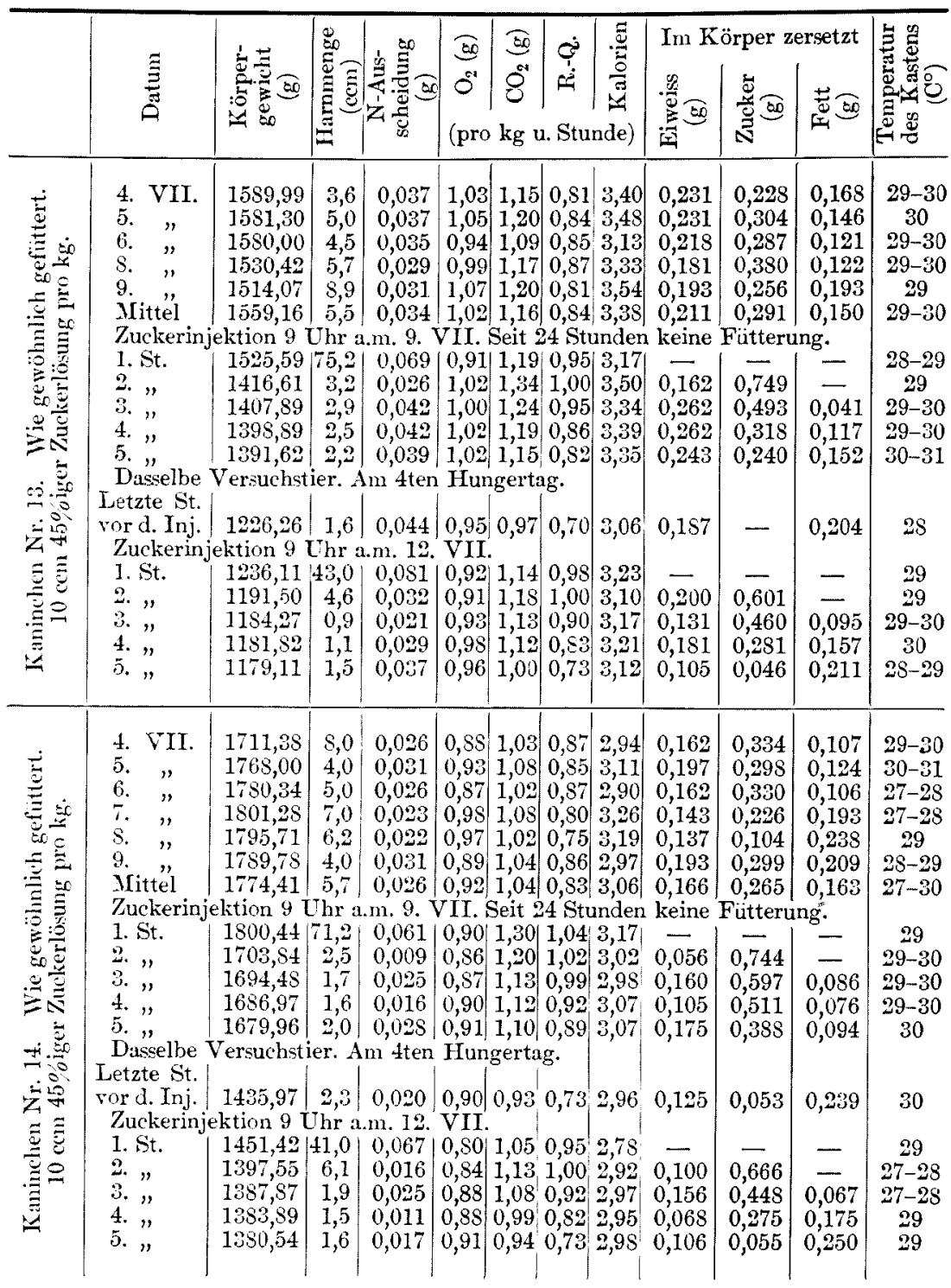

rührt sehr wahrscheinlich von der Differenz der infundierten Zuckermenge her. Im Folgenden seien meine diesbezüglichen Versuche in Kürze angeführt.

5, 25 resp. 45\% ${ }^{\circ}$ ige Lösung ron Traubenzucker (wasserfrei, M e rck) wurde zur Infusion verwendet. Die übrige Versuchsanordnung gleich wie bei den rorigen Versuchen. 


\begin{tabular}{|c|c|c|c|c|c|c|c|c|c|c|c|c|}
\hline & 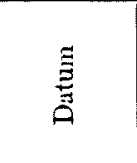 & 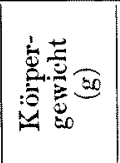 & 总 & 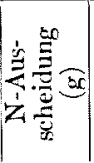 & $\begin{array}{c}\frac{80}{0} \\
\text { (pro }\end{array}$ & $\begin{array}{l}\frac{30}{80} \\
8 \\
8 \\
\operatorname{kg} u\end{array}$ & $\left|\begin{array}{l}\dot{q} \\
\dot{\sim}\end{array}\right|$ & 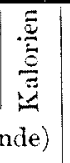 & 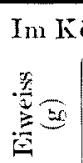 & 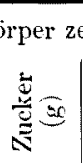 & 起 & 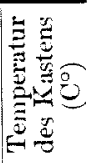 \\
\hline 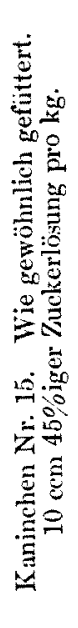 & 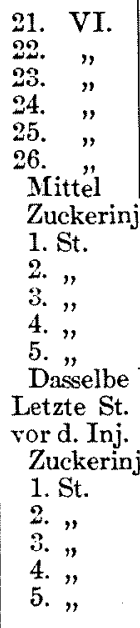 & 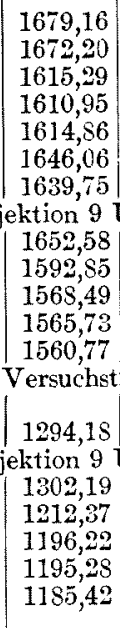 & 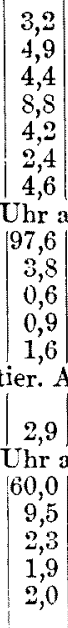 & $\begin{array}{c}0,026 \\
0,016 \\
0,022 \\
0,028 \\
0,022 \\
0,021 \\
0,022 \\
\mathrm{~m} .26 . \\
0,074 \\
0,009 \\
0,003 \\
0,009 \\
0,021\end{array}$ & \begin{tabular}{|l|}
0,94 \\
0,90 \\
0,55 \\
0,85 \\
0,92 \\
0,98 \\
0,90 \\
VI. \\
$\mid 0,90$ \\
0,95 \\
0,93 \\
0,92 \\
0,90
\end{tabular} & $\begin{array}{l}1,04 \\
1,10 \\
1,05 \\
1,02 \\
1,02 \\
1,09 \\
1,05 \\
\text { Seit } 2 \\
1,22 \\
1,33 \\
1,21 \\
1,16 \\
1,10\end{array}$ & $\begin{array}{l}0,80 \\
0,90 \\
0,92 \\
0,90 \\
0,80 \\
0,86 \\
0,86 \\
24 \mathrm{Stt} \\
0,98 \\
1,03 \\
0,95 \\
0,92 \\
0,89\end{array}$ & $\begin{array}{l}3,11 \\
3,05 \\
2,87 \\
2,86 \\
3,07 \\
3,13 \\
3,01 \\
\text { unden } \\
3,15 \\
3,34 \\
3,31 \\
3,17 \\
3,06\end{array}$ & $\begin{array}{c}0,164 \\
0,104 \\
0,140 \\
0,180 \\
0,139 \\
0,131 \\
0,143 \\
\text { keine } \\
-\overline{0} \\
0,056 \\
0,018 \\
0,056 \\
0,181\end{array}$ & $\begin{array}{c}0,206 \\
0,461 \\
0,444 \\
0,370 \\
0,212 \\
0,361 \\
0,342 \\
\text { Futteru } \\
-\overline{0}- \\
0,527 \\
0,719 \\
0,570 \\
0,417\end{array}$ & $\begin{array}{c}0,176 \\
0,095 \\
0,066 \\
0,076 \\
0,181 \\
0,130 \\
0,121 \\
\text { ng. } \\
- \\
-\overline{-} \\
0,059 \\
0,086 \\
0,101\end{array}$ & $\begin{array}{c}28-29 \\
28-29 \\
29 \\
29-30 \\
29 \\
29-30 \\
28-30 \\
\\
29 \\
29-30 \\
29-30 \\
29-30 \\
29\end{array}$ \\
\hline
\end{tabular}

Der R.-Q. wuchs nach Zuckerinjektion in allen Versuchen so beträchtlich an, dass er in einigen Stunden annähernd 1,0 betrug. Diese Erscheinung trat schon gleich nach Zuckerinfusion ein, exreichte aber ihren Maximalwert gewöhnlich 1-2 Stunden danach, um dann wicler zum Ausgangswert zurückzukehren. Dic Höhe und Dauer der R.-Q.-Steigerung stehen mit der Menge des injizierten Zuckers insofern in Beziehung, als sie um so grösser war und länger dauerte, je mehr Zucker dabei verwendet wurde. Diese Erhöhung des R.-Q. beruht in diesem Falle hauptsächlich auf der Zunahme der $\mathrm{CO}_{2}$-Produktion. Der $\mathrm{O}_{2}$ - Terbrauch war dabei in der Mehrzahl der Fälle etwas vermindert, sonst aber war eher das Umgekehrte der Fall; eine Schwankung, die wahrscheinlich innerhalb der Fehlergrenze liegt. Dieses Anwachsen des R.-Q. muss in diesen Falle wahrscheinlich auf die Verbrennung des infundierten Zuckers zurückzuführen sein. Die Sicherheit dieses Schlusses wird aber dadurch bedenklich, dass nach einigen modernsten Forschungsresultaten Zucker nur in einer besonderen aktiven, sog. alloiomorphen Form im Tierkörper angegriffen werden soll. Der infundierte Zucker gehört natürlich nicht zu dieser Form. Hicr liesse sich vielleicht der Einwand erheben, dass die Erhöhung des R.-Q. nach Zuckerinfusion nicht auf Verbrennung des infundierten Zuckers selbst beruhte, 
sondern von zufällig stattgefundener Hyperglykämie hervorgerufen wurde, die nach Infusion einer Lösung manchmal unspezifisch eintritt. Daher

\section{Tabelle VI.}

Grundumsatz nach Milchzuckerinfusion.

\begin{tabular}{|c|c|c|c|c|c|c|c|c|c|c|c|c|}
\hline & $\stackrel{\Xi}{\Xi}$ & $\underbrace{\frac{1}{5}}_{0}$ & 总 & $\frac{1}{2}$ & $\left\{\begin{array}{l}\frac{80}{8} \\
6 \\
\text { pro }\end{array}\right.$ & $\left|\begin{array}{l}\frac{30}{80} \\
8\end{array}\right|$ & $\begin{array}{l}\dot{\leftrightarrow} \\
\dot{\varepsilon}\end{array}$ & 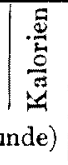 & $\begin{array}{l}\operatorname{Im} \mathrm{K} \\
\frac{3}{3} \sqrt[30]{3} \\
\frac{3}{3}\end{array}$ & 昰包 & $\stackrel{\vec{\theta}}{\theta 00}$ & 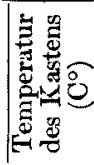 \\
\hline 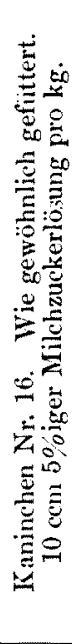 & 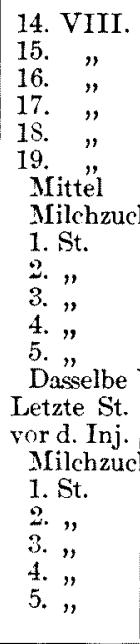 & \begin{tabular}{|l}
17 \\
16 \\
16 \\
16 \\
16 \\
16 \\
keri \\
17 \\
16 \\
16 \\
16 \\
16 \\
Ver
\end{tabular} & $\begin{array}{r}1,3 \\
5,5 \\
2,8 \\
2,0 \\
1,3 \\
2,6 \\
2,6 \\
\text { n } 9 \\
4,6 \\
2,4 \\
2,6 \\
2,3 \\
2,1 \\
\text { er. A }\end{array}$ & \begin{tabular}{|c|}
0,016 \\
0,022 \\
0,025 \\
0,021 \\
0,026 \\
0,026 \\
0,023 \\
Uhr a.n \\
$\left|\begin{array}{l}0,036 \\
0,026 \\
0,030 \\
0,019 \\
0,029\end{array}\right|$
\end{tabular} & $\begin{array}{l}0,96 \\
0,94 \\
0,91 \\
0,90 \\
0,90 \\
0,94 \\
0,92 \\
12.19 . \\
0,98 \\
0,94 \\
0,98 \\
0,91 \\
0,99\end{array}$ & $\begin{array}{l}1,11 \\
1,11 \\
1,10 \\
1,05 \\
1,09 \\
1,12 \\
1,10 \\
\text { VII } \\
1,14 \\
1,07 \\
1,14 \\
1,03 \\
1,05\end{array}$ & $\begin{array}{l}0,84 \\
0,86 \\
0,88 \\
0,85 \\
0,89 \\
0,87 \\
0,86 \\
\text { II. Se } \\
0,86 \\
0,82 \\
0,85 \\
0,85 \\
0,75\end{array}$ & $\begin{array}{l}3,23 \\
3,16 \\
3,09 \\
3,03 \\
3,08 \\
3,18 \\
3,13 \\
\text { eit } 24\end{array}$ & $\begin{array}{c}0,100 \\
0,137 \\
0,156 \\
0,131 \\
0,162 \\
0,162 \\
0,141 \\
\text { Stunde } \\
0,225 \\
0,162 \\
0,187 \\
0,118 \\
0,181\end{array}$ & $\begin{array}{l}0,341 \\
0,360 \\
0,383 \\
0,324 \\
0,393 \\
0,370 \\
0,362 \\
\text { keine } \\
0,322 \\
0,254 \\
0,324 \\
0,331 \\
0,099\end{array}$ & $\begin{array}{c}0,164 \\
0,132 \\
0,107 \\
0,136 \\
0,095 \\
0,119 \\
0,125 \\
\text { Fütter } \\
0,118 \\
0,162 \\
0,136 \\
0,139 \\
0,226\end{array}$ & \begin{tabular}{|c}
$29-3$ \\
$29-3$ \\
$28-2$ \\
29 \\
$29-3$ \\
$29-3$ \\
$28-3$ \\
ung. \\
29 \\
$30-3$ \\
31 \\
$30-3$ \\
$30-3$
\end{tabular} \\
\hline 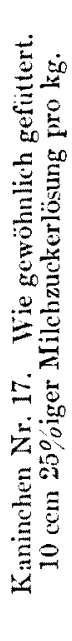 & 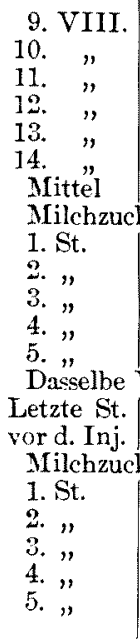 & $\begin{array}{l}17 \\
17 \\
17 \\
17 \\
16 \\
17\end{array}$ & \begin{tabular}{|r|}
5,0 \\
2,2 \\
3,2 \\
5,7 \\
2,6 \\
2,1 \\
2,1 \\
3,5 \\
on 9 \\
15,1 \\
2,1 \\
2,5 \\
2,2 \\
1,7
\end{tabular} & $\begin{array}{l}27 \\
25 \\
22 \\
25 \\
25 \\
14 \\
23\end{array}$ & $\begin{array}{l}0,94 \\
0,87 \\
0,83 \\
0,85 \\
0,88 \\
0,88 \\
0,87 \\
2.14 \\
0,84 \\
0,94 \\
0,86 \\
0,91 \\
0,94\end{array}$ & $\begin{array}{l}0,93 \\
0,97 \\
0,96 \\
1,03 \\
1,05 \\
0,99 \\
\text { VIII } \\
0,95 \\
1,02 \\
0,94 \\
1,01 \\
1,00\end{array}$ & $\begin{array}{l}0,78 \\
0,77 \\
0,81 \\
0,82 \\
0,86 \\
0,86 \\
0,82 \\
\mathrm{I} . \mathrm{Se} \\
0,83 \\
0,78 \\
0,80 \\
0,80 \\
0,76\end{array}$ & $\begin{array}{l}2,92 \\
2,72 \\
2,89 \\
3,00 \\
2,93\end{array}$ & $\begin{array}{l}0,168 \\
0,162 \\
0,150 \\
0,106 \\
0,175\end{array}$ & $\begin{array}{c}0,032 \\
0,048 \\
0,026 \\
- \\
-\end{array}$ & $\begin{array}{l}0,240 \\
0,242 \\
0,275 \\
0,235\end{array}$ & $\begin{array}{c}30-31 \\
29 \\
29-31 \\
30 \\
29 \\
29-31 \\
\mathrm{Ing} . \\
29 \\
30 \\
29-30 \\
30-31 \\
31\end{array}$ \\
\hline
\end{tabular}




\begin{tabular}{|c|c|c|c|c|c|c|c|c|c|c|c|c|}
\hline & $\stackrel{\bar{E}}{\bar{E}}$ & $\underset{0}{0}$ & 递 & $\frac{4}{3}$ & $\begin{array}{l}\begin{array}{c}80 \\
0^{2}\end{array} \\
\text { (pro }\end{array}$ & \begin{tabular}{|}
$\frac{80}{30}$ \\
8 \\
$\mathrm{gg} \mathrm{u}$
\end{tabular} & 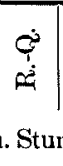 & 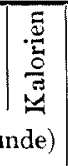 & 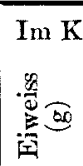 & 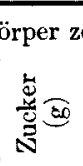 & $\underset{\Phi}{\vec{W}}$ & 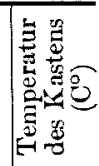 \\
\hline 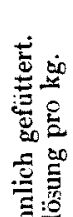 & $\begin{array}{l}\text { 13. VII. } \\
14 . " " \\
15 . " " \\
16 . " \text { " } \\
17 . \text { " } \\
\text { 1S. " } \\
\text { Mittel }\end{array}$ & $\begin{array}{l}1641,74 \\
1616,06 \\
1605,76 \\
1611,80 \\
1610,12 \\
1513,02 \\
1599,75\end{array}$ & $\begin{array}{l}3,3 \\
3,1 \\
3,2 \\
5,2 \\
2,9 \\
2,0 \\
3,3\end{array}$ & $\begin{array}{l}0,044 \\
0,035 \\
0,036 \\
0,043 \\
0,041 \\
0,039 \\
0,040\end{array}$ & $\begin{array}{l}0,90 \\
0,87 \\
0,93 \\
0,86 \\
0,90 \\
0,95 \\
0,90\end{array}$ & $\begin{array}{l}1,05 \\
1,03 \\
1,08 \\
1,02 \\
1,04 \\
1,09 \\
1,05\end{array}$ & $\begin{array}{l}0,87 \\
0,88 \\
0,86 \\
0,90 \\
0,85 \\
0,85 \\
0,87\end{array}$ & $\begin{array}{l}2,97 \\
2,91 \\
3,10 \\
2,86 \\
2,98 \\
3,13 \\
2,99\end{array}$ & $\begin{array}{l}0,275 \\
0,218 \\
0,225 \\
0,268 \\
0,256 \\
0,243 \\
0,247\end{array}$ & $\begin{array}{l}0,269 \\
0,312 \\
0,299 \\
0,302 \\
0,248 \\
0,274 \\
0,284\end{array}$ & $\begin{array}{l}0,086 \\
0,087 \\
0,109 \\
0,063 \\
0,104 \\
0,104 \\
0,092\end{array}$ & $\begin{array}{l}28-29 \\
28-29 \\
29-30 \\
29-31 \\
30-31 \\
30-31 \\
28-31\end{array}$ \\
\hline 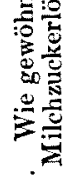 & $\begin{array}{l}\text { Milch } \\
\text { 1. St. } \\
\text { 2. " } \\
\text { 3. " } \\
\text { 4. " } \\
\text { 5." }\end{array}$ & $\begin{array}{l}15 \\
14 \\
14 \\
14 \\
14\end{array}$ & $\begin{array}{r}46,2 \\
4,4 \\
2,8 \\
1,8 \\
2,4\end{array}$ & $\begin{array}{l}0, \\
0, \\
0, \\
0, \\
0,\end{array}$ & $\mid \begin{array}{l}18 . \\
0,90 \\
0,94 \\
0,88 \\
0,89 \\
0,95\end{array}$ & $\begin{array}{l}\text { VII } \\
1,07 \\
1,10 \\
1,00 \\
1,06\end{array}$ & $\mid \begin{array}{l}\text { Seit } \\
0,86 \\
0,85 \\
0,82 \\
0,88 \\
0,81\end{array}$ & \begin{tabular}{|l|l|} 
& $24 \mathrm{~S}$ \\
3,06 \\
3,16 \\
2,96 \\
3,00 \\
3,12
\end{tabular} & $\begin{array}{c}\text { tunden } \\
0,081 \\
0,093 \\
0,175 \\
0,300\end{array}$ & $\begin{array}{c}\text { keine } \\
- \\
0,371 \\
0,264 \\
0,355 \\
0,173\end{array}$ & $\begin{array}{c}- \\
0,153 \\
0,168 \\
0,098 \\
0,127\end{array}$ & $\begin{array}{c}\text { g. } \\
31 \\
31 \\
31 \\
31-32 \\
31-32\end{array}$ \\
\hline 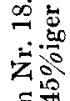 & $\begin{array}{l}\text { Letzte St. } \\
\text { vord. Inj. } \\
\text { Milchzuc }\end{array}$ & & $\begin{array}{r}2,6 \\
\text { ion } 9\end{array}$ & $\begin{array}{l}0 \\
\mathbf{U} h\end{array}$ & $\begin{array}{l}0,90 \\
\text { ก. } 21 .\end{array}$ & 1 & 0,72 & 2,92 & 0,218 & 0,025 & 0,205 & 29 \\
\hline & 1. St. & 121 & 29,5 & & 0,93 & & {$[0,77 \mid$} & \begin{tabular}{l|l}
7 & 3,09 \\
\end{tabular} & - & $\ldots$ & & 30 \\
\hline & & & 6 & & & & 0 & 12,9 & & & & 31 \\
\hline & $\begin{array}{l}3 \\
4 .\end{array}$ & & 3,0 & & $\begin{array}{l}0,91 \\
0,93\end{array}$ & & $\mid \begin{array}{l}0,73 \\
0,72\end{array}$ & \begin{tabular}{l|l}
3 & 2,96 \\
3 & 304
\end{tabular} & $\begin{array}{l}0,225 \\
0,175\end{array}$ & $\begin{array}{l}0, \\
0,\end{array}$ & $\begin{array}{l}0,197 \\
0,234\end{array}$ & $\begin{array}{r}31 \\
30-3\end{array}$ \\
\hline & & 110 & 1,5 & 0,029 & 0,91 & & & $\begin{array}{l}3,94 \\
2,94\end{array}$ & 0,181 & 0,047 & 0,215 & 31 \\
\hline
\end{tabular}

habe ich, um darüber was Sicheres zu erfahren, eine andere Versuchsreihe angestellt, in der Milchzuckerlösungen, von ann̈̈hernd gleichem C-Gehalt wie dem der in den früheren Versuchen angewandten Glukoselösungen, unter sonst gleicher Versuchsanordnung den Versuchstieren injiziert wurden. Dass intravenös eingef ührter Milchzucker fast unverändert wieder im Harn erscheint, war schon durch $\mathrm{Pavy},{ }^{13)} \mathrm{Voit},{ }^{14)}$ Blumenthal, ${ }^{15)}$ Klapp ${ }^{16)}$ Schlayer u. 'Takay asu ${ }^{17)}$ u. a. bekannt.

Wie aus den Tabellen zu ersehen ist, blieb der R.-Q. nach Milchzuckerinfusion fast immer unverändert. Danach kann man ohne weiteres annehmen, dass das Anwachsen des R.-Q. nach Traubenzuckerinfusion wirklich von der Verbrennung der einverleibten Substanz selbst herrïhrt. Bemerkenswert ist noch, dass die Fettzersetzung, die bei Hunger besonders lebhaft vor sich ging, nach Glukoseinfusion beträchtlich gemildert wurde.

Noch etwas ist uns bei diesen Zuckerversuchen besonders anfgefallen. Intra venös injizierter Zucker wirkte ausgesprochen diuretisch, besonders wenn er in hypertonischer Lösung gegeben wurde, was sehr wahrscheinlich durch reichliche Zuckerausscheidung bewirkt wird. Diese Harnflut bringt gleichzeitig reichliche $\mathrm{N}$-A usscheidung mit sich, eine Erscheinung, die viele Autoren mit dem Namen „Ausschwemmung“ belegen. Die Frage bleibt 
noch unentschieden, ob sie auf der Ausschwemnung des präformierten Schlack- $N$ beruht, oder ob das Körpereiweiss dabei übermässig zersetzt wird. Hier habe ich bei Berechnung der im Körper zersetzten Substanzmenge die Spalten in der ersten Stunde nach Zuckerinfusion absichtlich unberührt gelassen, da in dieser Stunde die N-Ausscheidung so gross war, dass man dabei kaum einen wirklichen Eiweisszerfall annehmen kann.

\section{Zusammenfassung.}

1. Intravenöse Fettzufuhr bewirkt keine spezifisch-dynamische Stoffwechselsteigerung.

2. Nüchternen Tieren eingespritzt, führt Fett keine Schwankung des Respirationsquotienten herbei, im Gegensatz zu Traubenzucker, der sofort dessen Steigerung hervorruft.

3. Der Kraftwechsel eines mässig gehungert habenden 'Tiers wird auch durch direkte Fettzufuhr in seinem Modus nicht verändert, solange es noch keinen Mangel an genügendem Fettvorrat leidet.

4. Erst bei fortschreitender Karenz, bei der der umsetzbare Fettvorrat erschöpft wird, ist die sofortige Verbrennung des infundierten Fetts auffallend bemerkbar, wodurch das Körpereiweiss vor übermüssiger Zersetzung bewahrt bleibt.

\section{Literatur.}

(1) v. Leube, W., Deutsche Klinik, 1901, 1, 71.

(2) Ja cob, P., Kongr. f. inn. Med., 1898, 16, 135.

(3) Du Mesnil de Rochemont, Dtsch. Arch. f. kl. Med., 1S98, 60, 474.

(4) Strauss, H., Ztralbl. f. inn. Med., 1899, 20, 213.

(5) Winternitz, H., Zts. f. kl. Med., 1903, 50, 80 .

(6) Heilner, E., Zts. f. Biol, 1910, 54, 54.

(7) Mills, L. H. u. Murlin, J. R., Proc. of Soc. Exp. Biolog. \& Med., 1909-10, 7, 166.

(8) Haldane, J., J. Physiol., 1892, 13, 419.

(9) Zuntz u.Schumberg, Endocrinology and Metadolism, New York 1922, 3, 565.

(10) Marine, D., J. Metab. Research, 1922, 2, 329.

(11) Falta, B. u. Bernstein, S., Wien. kl. Woch. 1924, 635 ; 1924, 1423; Wien. med. Woch., 1912, 2379.

(12) Verzàr, F., Bioch. Zts., 1911, 34, 63.

(13) Par y, F. W., J. Physiol., 1899, 24, 479.

(14) Voit, F., Arch. f. kl. Med., 1897, 58, 545.

(15) Blumenthal, F., Hofmeister's Beitrïge, 1905, 6, 329.

(16) K la p p, R., Mitteil. a. d. Grenzgeb. d. Med. u. Chir., 1902, 10, 254.

(17) Schlayeru. Takayasu, R., Dtsch. Arch. f. kl. Med. 1910, 98, 17. 